\title{
MicroRNA-326 suppresses the proliferation, migration and invasion of cervical cancer cells by targeting ELK1
}

\author{
PING ZHANG ${ }^{1}$, FENG KONG ${ }^{2}$, XINCHAO DENG ${ }^{1}$, YUNHAI YU ${ }^{1}$, \\ CONGZHE HOU ${ }^{1}$, TINGTING LIANG ${ }^{1}$ and LIN ZHU $^{1}$ \\ ${ }^{1}$ Department of Gynecology; ${ }^{2}$ Central Laboratory, \\ The Second Hospital of Shandong University, Jinan, Shandong 250033, P.R. China
}

Received July 24, 2015; Accepted January 12, 2017

DOI: $10.3892 / \mathrm{ol} .2017 .5852$

\begin{abstract}
Although microRNAs (miRNAs or miRs) are able to function as oncogenes or tumor suppressors, the role of miR-326 in regulating human cervical cancer cells remains unclear. In the present study, the expression of miR-326 was identified to be downregulated in cervical cancer cell lines and primary tumor samples, and the overexpression of miR-326 decreased cell proliferation, migration and invasion in cervical cell lines. Bioinformatics prediction and experimental validation results revealed that the function of miR-326 was achieved by targeting and repressing ETS domain-containing protein Elk-1 (ELK1) expression. ELK1 was targeted directly by miR-326, which was downregulated in human cervical cancer tissues compared with that in adjacent normal tissues. The results of the present study suggest that miR-326, a potential tumor suppressor, may be used in the treatment of cervical cancer.
\end{abstract}

\section{Introduction}

Cervical carcinoma is the second most common cause of cancer mortality in females following breast cancer and seriously affects the health of females worldwide, particularly in developing countries (1-3). Cervical cancer is caused by the abnormal expression of certain oncogenes and tumor-suppressor genes. It has been reported that a number of genes, including Bcl-2-associated $X$ and $p 53$, serve important roles in cervical cancer progression (4). In addition to functional genes, noncoding RNAs, including microRNAs (miRNAs or miRs), may serve a role in cervical cancer (5).

miRNAs were identified in the early 1990s and were recognized to serve an important role in tumorigenesis (6).

Correspondence to: Professor Lin Zhu, Department of Gynecology, The Second Hospital of Shandong University, 247 Beiyuan Road, Jinan, Shandong 250033, P.R. China

E-mail: sdey_zhulin2015@163.com

Key words: microRNA-326, ETS domain-containing protein Elk-1, cervical cancer
miRNAs modulate gene expression through messenger RNA (mRNA) degradation and/or translational repression at the post-transcriptional level (7). miRNAs also mediate translational repression and/or mRNA degradation by binding to the 3'-untranslated regions (3'-UTRs) of their target mRNAs (8). A number of miRNAs have been demonstrated to serve an important role in numerous cellular processes, including cell differentiation, proliferation and apoptosis, which are all involved in tumor development (9). Abnormal expression levels of miRNAs are associated with various tumor types, including hepatocellular carcinoma, cervical cancer, ovarian carcinoma, colorectal cancer and breast cancer (10-14). miRNAs are able to act either as oncogenes or as tumor suppressors by regulating the expression of their target genes (15). Therefore, investigation of aberrant miRNA expression may identify novel miRNA biomarkers for cervical carcinoma.

miR-326 was initially identified in neurons and reported to be upregulated in zebrafish embryos treated with the Notch pathway inhibitor $(16,17)$. Previous studies suggested that the dysregulation of miR-326 contributed to various types of cancer, including medulloblastoma, chronic lymphocytic leukemia, pancreatic ductal adenocarcinoma and cholangiocarcinoma (18-22). The expression of miR-326 was downregulated in gliomas, and transfection with miR-326 markedly reduced the occurrence of tumors in glioma cells in vivo (23). Zhou et al (24) identified that miR-326 served an important role in human glioma by targeting Nin one-binding protein and mitogen-activated protein kinase (MAPK) signaling pathway activity.

In the present study, miR-326 was identified to be downregulated in cervical cancer, and its overexpression suppressed the proliferation, migration and invasion of cervical cancer cells in vitro. Furthermore, miR-326 inhibited the expression of ETS domain-containing protein Elk-1 (ELK1) at the mRNA and protein levels. Therefore, miR-326 was identified to function as a tumor suppressor by targeting ELK1. The results of the present study provide a basis for the use of miR-326 as a tumor suppressor in the treatment of cervical cancer.

\section{Materials and methods}

Cell culture and transfection. The cervical cancer cell lines CaSki and HeLa were purchased from the Cell Bank of Type 
Culture Collection of the Chinese Academy of Sciences (Shanghai, China). Cells were cultured in RPMI 1640 medium (Cambrex, Walkersville, MD, USA) supplemented with $10 \%$ fetal bovine serum (FBS; HyClone; GE Healthcare Life Science, Logan, UT) at $37^{\circ} \mathrm{C}$ and $5 \% \mathrm{CO}_{2}$. Transfection of cells with miR-326 was carried out using Lipofectamine ${ }^{\mathrm{TM}} 2000$ reagent (Invitrogen; Thermo Fisher Scientific, Inc., Waltham, MA, USA), according to the manufacturer's protocol.

Clinical specimens. A total of 25 primary cervical cancer specimens and 25 non-cancerous specimens were collected from female patients who had undergone surgical treatment at The Second Hospital of Shandong University (Jinan, China) between January 2014 and September 2014. The mean of the patients was 47 years (range, 40-55 years). The samples were processed (liquid nitrogen frozen) and stored in RNAlater (Qiagen, Inc., Valencia, CA, USA) at $-20^{\circ} \mathrm{C}$ prior to extraction of RNA. The present study was approved by the Bioethics Committee of Shandong University (Jinan, China), and written informed consent and approval were provided by each patient prior to the study.

Reverse transcription-quantitative polymerase chain reaction (RT-qPCR). RNA was isolated from tissue and cell samples using TRIzol ${ }^{\circledR}$ (Invitrogen; Thermo Fisher Scientific, Inc.), according to the manufacturer's protocol. A polyA tail was added to the mature miRNA template, and complementary DNA (cDNA) was synthesized using a polyT primer with a 3'degenerate anchor and a 5' universal tag. RT-qPCR was carried out using a miRCURY LNA ${ }^{\mathrm{TM}}$ Universal RT microRNA PCR system with SYBR Green master mix and LNA-based primer sets for target miRNAs (Homo sapiens-miR-326; cat. no. 204512) and U6 small nuclear RNA (cat. no. 203907; all from Exiqon, Inc., Woburn, MA, USA). PCR cycles were as follows: $94^{\circ} \mathrm{C}$ for $3 \mathrm{~min}$, followed by 40 cycles of $94^{\circ} \mathrm{C}$ for $30 \mathrm{sec}, 60^{\circ} \mathrm{C}$ for $30 \mathrm{sec}$ and $72^{\circ} \mathrm{C}$ for $30 \mathrm{sec}$. The relative levels of miRNAs in the cells and tissues were normalized to U6 and were calculated using the $2^{-\Delta \Delta \mathrm{Cq}}$ method (25). To quantify the level of ELK1 mRNA expression, $1 \mu \mathrm{g}$ total RNA was reverse transcribed to cDNA using PrimeScript RT reagent kit (Takara Biotechnology Co., Ltd., Dalian, China), and qPCR was carried out using the reverse-transcribed product, SYBR Green dye mix (Invitrogen; Thermo Fisher Scientific, Inc.) and specific primers for ELK1 and GAPDH, which were as follows: ELK1, 5'-CCTTCTATCAGCGTGGAT-3' (forward) and 5'-GTGGTG GTGGTAGTAGTC-3' (reverse); and GAPDH, 5'-ACCCAG AAGACTGTGGATGG-3' (forward) and 5'-CAGTGAGCT TCCCGTTCAG-3' (reverse). The thermo cycling conditions for the RT-qPCR were as follows: $95^{\circ} \mathrm{C} 10 \mathrm{~min}, 40$ cycles of $95^{\circ} \mathrm{C}$ for $20 \mathrm{sec}, 55^{\circ} \mathrm{C}$ for $30 \mathrm{sec}, 72^{\circ} \mathrm{C}$ for $20 \mathrm{sec}$. The relative level was calculated using the $2^{-\Delta \Delta \mathrm{Cq}}$ and the level of ELKI mRNA was normalized to that of GAPDH.

Western blot analysis. Proteins were extracted using RIPA lysis buffer (Beyotime Institute of Biotechnology, Shanghai, China) and the concentration was measured using the Bio-Rad protein assay kit (Beijing Solarbio Science \& Technology Co., Ltd., Beijing, China) according to the manufacture's protocol. A total of $30 \mu \mathrm{g}$ protein was separated using $12.5 \%$ SDSPAGE, and then transferred onto polyvinylidene difluoride membranes. The membranes were blocked with 5\% non-fat milk at room temperature for $1 \mathrm{~h}$ and incubated with primary antibodies against ELK1 (cat. no. 14507-1-AP; dilution, 1:1,000; Proteintech) and GAPDH (Santa Cruz Biotechnology, Inc., Dallas, TX, USA) at $4^{\circ} \mathrm{C}$ overnight. The membranes were then incubated with horseradish peroxidase-conjugated secondary antibodies (cat. no. ab6721; dilution, 1:2,000; Abcam). The blots were examined using an enhanced chemiluminescence detection system according to the manufacture's protocol (Pierce; Thermo Fisher Scientific, Inc.). The band intensities of the western blotting were analyzed using Image Analysis Software v2.0 (Thermo Fisher Scientific, Inc.).

Cell proliferation assay. MTT and 5-ethynyl-2'-deoxyuridine (EdU) incorporation assays were used to determine cell proliferation. Cells were seeded into 96 -well plates at $4 \times 10^{4}$ cells/well in triplicate for each transfection group: miR-326 mimic, miR-326 inhibitor and blank control. MTT was added in each well $(5 \mathrm{mg} / \mathrm{ml})$ at various time points $(24,48$ and $72 \mathrm{~h})$ and then incubated in the dark at $37^{\circ} \mathrm{C}$ for $2 \mathrm{~h}$. Absorbance was determined at a wavelength of $570 \mathrm{~nm}$. An EdU incorporation assay was performed to analyze cell proliferation using a CellLight $^{\mathrm{TM}}$ EdU Apollo ${ }^{\circledR} 488$ In Vitro Imaging kit (Guangzhou RiboBio Co., Ltd., Guangzhou, China), according to the manufacturer's protocol.

Wound healing assay. Cells $\left(5 \times 10^{5}\right.$ cells $\left./ \mathrm{ml}\right)$ were seeded in 6-well plates and cultured in complete medium (RPMI-1640 medium with $10 \%$ FBS). Following 24 h of culture, when the cells had reached $90-100 \%$ confluence, a single wound was created in the center of the well by removing the attached cells with a sterile $200-\mu 1$ pipette tip. After 56 h of culture, cells that had migrated into the wounded area were visualized and images were captured under an inverted microscope. Each experiment was carried out at least three times independently. Wound closure was monitored over time and the percentage of closure was determined.

Invasion assay. A Matrigel invasion chamber was used to determine cell invasive capacity. A cell suspension $\left(5 \times 10^{5}\right.$ cells $\left./ \mathrm{ml}\right)$ was prepared in serum-free medium (RPMI-1640 medium with $0.1 \%$ FBS) for the transfected CaSki cells (transfected with miR-326 mimic, inhibitor or NC). A 300- $\mu$ l volume of cell suspension was added into the upper chamber coated with Matrigel, and $500 \mu \mathrm{l}$ of Dulbecco's modified Eagle's medium (Gibco; Thermo Fisher Scientific, Inc.) supplemented with $10 \%$ FBS was added into the lower chamber. The cells were then incubated at $37^{\circ} \mathrm{C}$ with $5 \% \mathrm{CO}_{2}$ for $24 \mathrm{~h}$. Cells on the upper surface were removed, whereas those on the lower surface were stained by $1 \%$ eosin for $30 \mathrm{~min}$. The cell number was determined in at least five randomly selected fields under a microscope (SZ61; Olympus, Tokyo, Japan).

Bioinformatics method. The following online miRNA target prediction algorithms were used to evaluate the potential target genes of miR-326: PicTar (http://www.pictar. org/), TargetScan (http://www.targetscan.org/vert_71/), Microcosm Targets (http://www.ebi.ac.uk/enright-srv/microcosm/htdocs/targets/v5/) and miRanda (http://www.microrna. org/microrna/home.do). 
Luciferase miRNA-binding assay. CaSki cells in a 96-well plate were transfected with $50 \mathrm{nM}$ miR-326 mimic or negative control miRNA and then co-transfected with the wild-type or mutant 3'-UTR of the ELK1 gene with $0.2 \mathrm{mg} / \mathrm{ml}$ vector (Ambion; Thermo Fisher Scientific, Inc.). Following $48 \mathrm{~h}$ of transfection, luciferase activity was determined using the Dual-Luciferase ${ }^{\circledR}$ Reporter Assay System (Promega Corporation, Madison, WI, USA). Firefly luciferase activity was then normalized to the corresponding Renilla luciferase activity. Luciferase assays were repeated in three independent experiments.

Overexpression of target gene ELK1. In order to construct ELK1 overexpressing plasmid, the gene without 3'-UTR was amplified from cDNA of human normal cervical tissues by polymerase chain reaction. The sequences of primers for cloning were: F, CTAGCTAGCATGGACCCATCTGTGACG CTGTG; R, CCGCTCGAGTCATGGCTTCTGGGGCCCT. The PCR products were cloned into the pMD18-T vector (TAKARA Biotechnology Co., Ltd.). Then, pCMV6-ELK1 was obtained by subcloning a NheI-XhoI fragment from pMD18-T/ELK1 into the NheI-XhoI site of pCMV6-Entry Vector.

Statistical analysis. Results are presented as the mean \pm standard deviation of three independent experiments. A Student's $t$ test was used to compare the experimental groups with the controls, whereas two-way analysis of variance followed with Bonferroni's multiple comparison test was used to determine the differences among three or more experimental groups. $\mathrm{P}<0.05$ was considered to indicate a statistically significant difference. SPSS 18.0 software (SPSS Inc., Chicago, IL, USA) was used to analyze the data.

\section{Results}

miR-326 is significantly downregulated in cervical cancer tissues and cell lines. miR-326 expression was determined in 25 fresh cervical cancer tissue specimens and 25 healthy control specimens. The results of RT-qPCR demonstrated that miR-326 expression was downregulated in cervical cancer tissues compared with that in non-neoplastic samples $(\mathrm{P}=0.0109$; Fig. 1). Significantly decreased miR-326 expression was also demonstrated in cervical cancer cell lines (HeLa, $\mathrm{P}=0.0316$; and CaSki, $\mathrm{P}=0.0045$; Fig. 1) using RT-qPCR. In spite of the variation in the degree of downregulation of miR-326 being observed in the cell lines, the expression was relatively low. As downregulation was more evident in the CaSki cell line than in the HeLa cell line, CaSki cells were used for subsequent experiments.

miR-326 inhibits the proliferation, migration and invasion of human cervical cells. As miR-326 expression was significantly downregulated in cervical cancer tissues, it was hypothesized that it may be involved in tumor cell proliferation. Therefore, the effect of the overexpression or low expression of miR-326 on the proliferation of cervical cancer cell lines (CaSki and HeLa) was investigated. The results of an MTT assay revealed that transfection with miR-326 mimic inhibited the proliferation of cervical cancer cells (Fig. 2A, Caski: $\mathrm{P}_{24 \mathrm{~h}}=0.0416$;

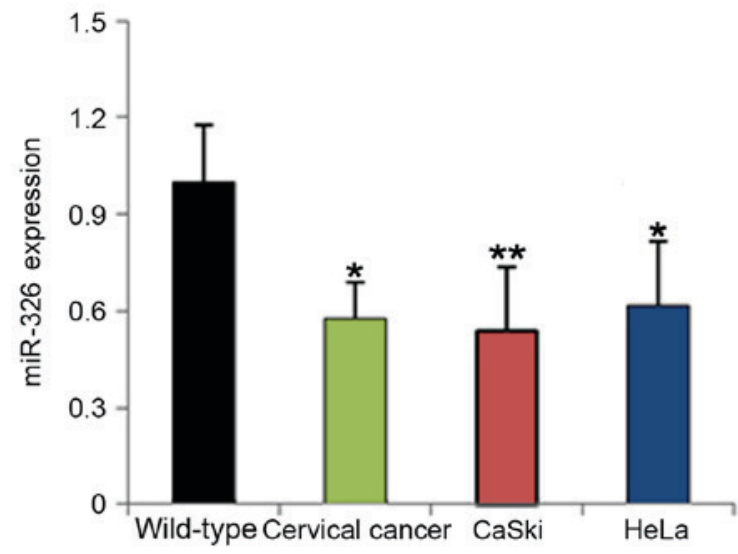

Figure 1. Expression levels of miR-326 in clinical specimens $\left({ }^{* *} \mathrm{P}<0.01\right.$ vs. wild-type) and cervical cancer cell lines (CaSki and HeLa) relative to wild-type cervical tissue $\left({ }^{* *} \mathrm{P}<0.01 ;{ }^{*} \mathrm{P}<0.05\right.$ vs. wild-type). The reverse transcription-quantitative polymerase chain reaction demonstrated that the expression levels of miR-326 were significantly lower in cervical cancer tissues and cell lines than those in wild-type cervical tissues. U6 was used as an internal control. miR, microRNA.

$\mathrm{P}_{48 \mathrm{~h}}=0.0356 ; \mathrm{P}_{72 \mathrm{~h}}=0.0528 ;$ Hela: $\mathrm{P}_{24 \mathrm{~h}}=0.00245 ; \mathrm{P}_{48 \mathrm{~h}}=0.00986$; $\left.\mathrm{P}_{72 \mathrm{~h}}=0.0238\right)$. Cervical cancer cells transfected with the miR-326 inhibitor demonstrated enhanced cell proliferation compared with that of the control (Fig. 2A, Caski: $\mathrm{P}_{24 \mathrm{~h}}=0.0969$; $\mathrm{P}_{48 \mathrm{~h}}=0.00221 ; \mathrm{P}_{72 \mathrm{~h}}=0.0055$; Hela: $\mathrm{P}_{24 \mathrm{~h}}=0.011 ; \mathrm{P}_{48 \mathrm{~h}}=0.0104$; $\mathrm{P}_{72 \mathrm{~h}}=0.0841$ ), which was similar to the results obtained using an EdU incorporation assay (Fig. 2B). These results indicated that miR-326 serves an important role in suppressing cervical cancer proliferation.

Migration and invasion assays were carried out to investigate the effects of miR-326 on cervical cancer cells in vitro. Cervical cancer cells in the miR-326 mimic group exhibited decreased migratory and invasive abilities compared with those displayed by control cells (Fig. 2C). Conversely, the miR-326 inhibitor promoted the migration and invasion of cervical cells (Fig. 2C). These effects were determined to be statistically significant in each cell type (Fig. 2D).

Identification of candidate genes targeted by miR-326. To reveal the molecular mechanism underlying the inhibition of the proliferation and metastasis of cervical cancer cells by miR-326, a combination of four bioinformatics algorithms was used (TargetScan, PicTar, miRanda and Microcosm Targets) to search for accurate potential targets of miR-326. All four approaches predicted $E L K 1$ as a potential target of miR-326, and the 3'-UTR of ELK1 mRNA contained a highly conserved binding site between position 1,261 and position 1,268 of the miR-326 seed sequence (the core sequence that encompasses the first $2-8$ bases of the mature miRNA; Fig. 3A). The wild-type and mutant ELK1 3'-UTRs were generated with sequences presented in Fig. 3A. A dual-luciferase reporter assay was performed on CaSki cells, and luciferase activity was significantly decreased in CaSki cells co-transfected with the wild-type ELK1 3'-UTR and miR-326 mimic ( $\mathrm{P}=0.0010$; Fig. 3B). This result indicates that miR-326 directly targets the 3'-UTR of ELK1 in CaSki cells.

To investigate the association between miR-326 and ELK1 in human cervical cancer, ELK1 expression was determined 


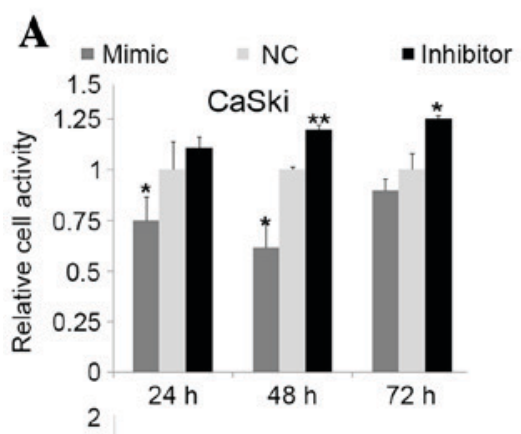

B
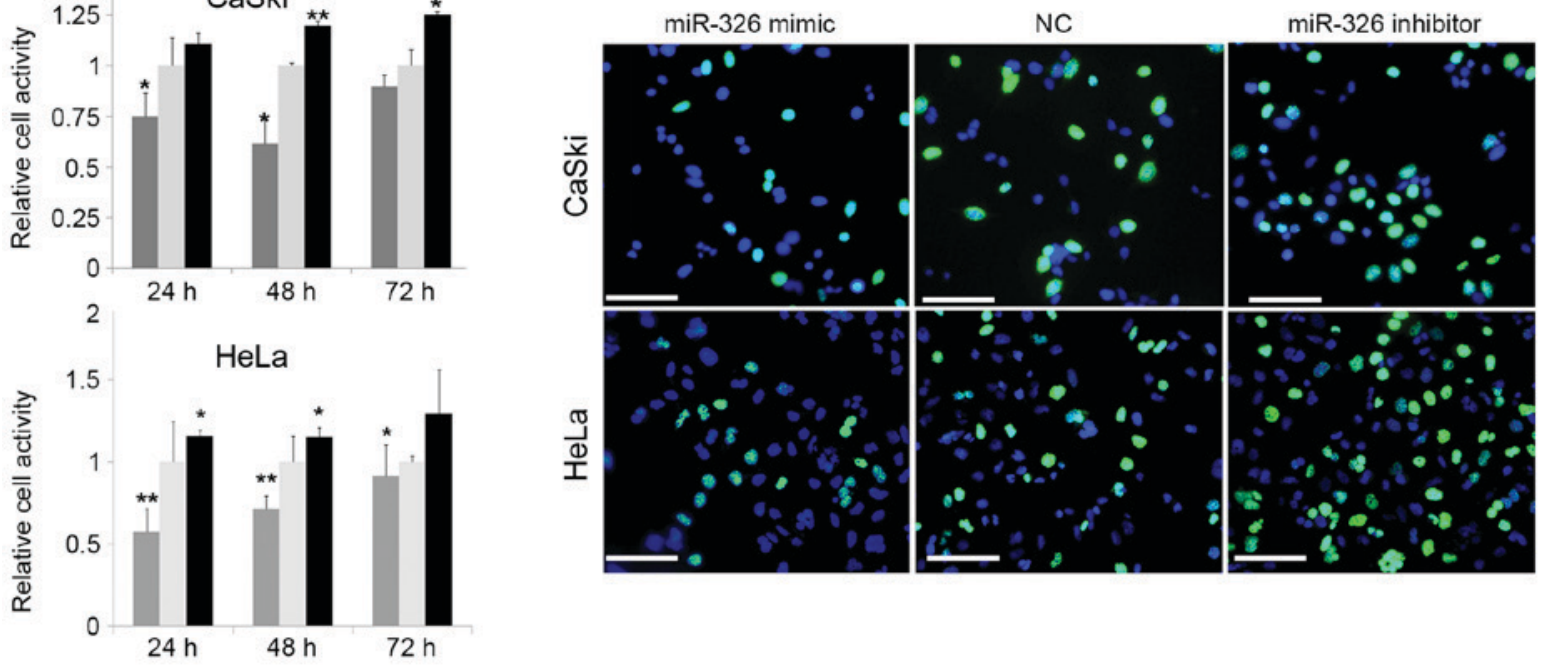

- miR-326 mimic aNC $\square$ miR-326 inhibitor

C Mimic NC Inhibitor
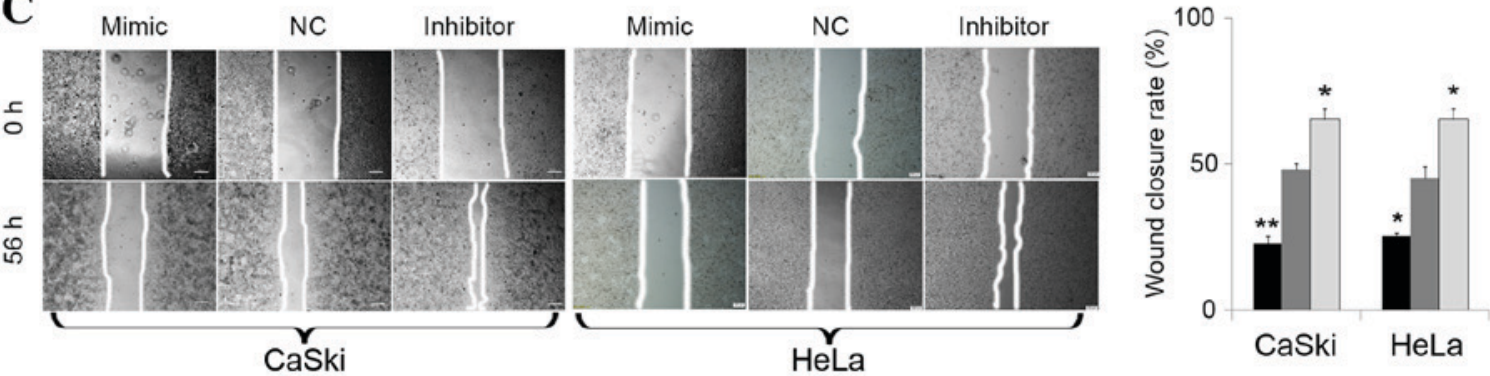

D
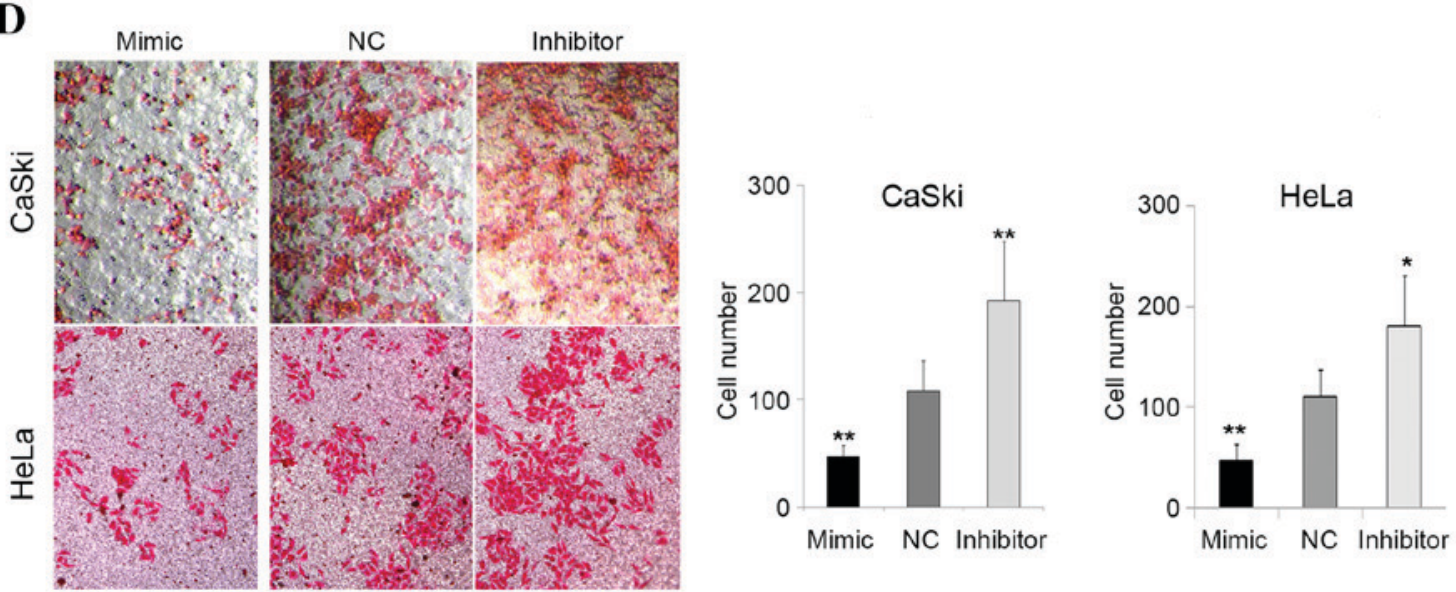

Figure 2. miR-326 suppresses cell proliferation. (A) MTT assay. CaSki and HeLa cells were transfected with miR-326 mimic (Caski: ${ }^{*} \mathrm{P}_{24 \mathrm{~h}}<0.05$; ${ }^{*} \mathrm{P}_{48 \mathrm{~h}}<0.05$ vs. NC; Hela: ${ }^{* *} \mathrm{P}_{24 h}<0.01 ;{ }^{* *} \mathrm{P}_{48 h}<0.01 ;{ }^{*} \mathrm{P}_{72 h}<0.05$ vs. NC), miR-326 inhibitor (Caski: ${ }^{* *} \mathrm{P}_{48 h}<0.01 ;{ }^{*} \mathrm{P}_{72 h}<0.05$ vs. NC; Hela: ${ }^{*} \mathrm{P}_{24 h}<0.05$; ${ }^{*} \mathrm{P}_{48 h}<0.05$ vs. NC) or scrambled miRNA (NC). Cell activity was quantified by measuring the absorbance at $570 \mathrm{~nm}$ and is presented relative to NC. (B) 5-Ethynyl-2'-deoxyuridine labeling (green) was performed following transfection of the two cell lines with miR-326 mimic, miR-326 inhibitor or NC. Nuclei were labeled with DAPI (blue). Bar, $100 \mu \mathrm{m}$. (C) A scratch wound-healing assay was conducted in CaSki and HeLa cells transfected with miR-326 mimic, miR-326 inhibitor or NC. The migration distance was measured at 0 and $56 \mathrm{~h}$ following scratching of the cells. Magnification, $\mathrm{x} 40$. (D) A Transwell assay revealed the invasion capacity of CaSki and HeLa cells transfected with miR-326 mimic, miR-326 inhibitor or NC. Cells were stained by $1 \%$ eosin. Magnification, x100. Average number of invading cells from three independent experiments. miR/miRNA, microRNA; NC, negative control.

in 25 cervical cancer tissues and 25 wild-type cervical tissues using RT-qPCR. ELK1 mRNA levels in cervical cancer tissues were significantly increased $(\mathrm{P}=0.0125$; Fig. $3 \mathrm{C})$ compared with those in wild-type cervical samples. The upregulation of ELK1 was associated with the downregulated expression of miR-326 in 25 cervical cancer tissues (Fig. 1). Therefore, the downregulation of miR-326 may contribute to the overexpression of ELK1 in human cervical cancer.

ELK1 expression is regulated by $\mathrm{miR}-326$ and inversely associated with miR-326 level in cervical cancer tissues. The role of miR-326 in the regulation of ELK1 mRNA expression levels 
A

Wild-type ELK1 3' UTR UUUCUAAAGAGUCCCAGAGA
miR-326 GACCUCCUUCCCGGGUCUCC
Mutant ELK1 3' UTR UUUCUAAAGAGUAACCGCGA

B

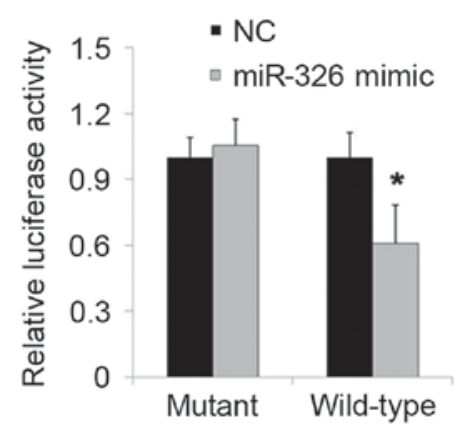

C

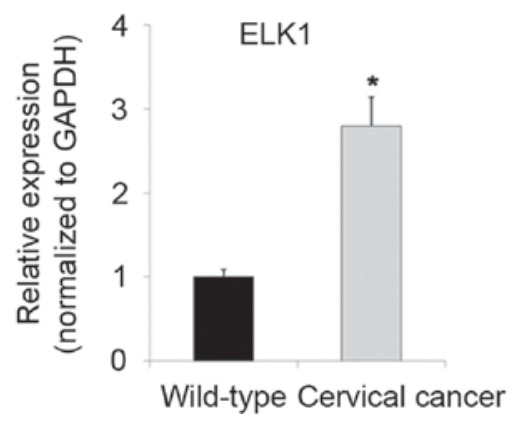

Figure 3. ELK1 is a direct target of miR-326. (A) Seed sequences of miR-326 against the wild-type or mutant 3'-UTR of ELK1. (B) A luciferase reporter assay identified that co-transfection of CaSki cells with miR-326 mimic and wild-type ELK1 3'-UTR led to a significant decrease in luciferase activity compared with co-transfection with scrambled miRNA (NC) and wildtype ELK1 3'-UTR ('P<0.05). By contrast, co-transfection with the mutant ELK1 3'-UTR and miR-326 mimic demonstrated no significant difference compared with co-transfection with mutant ELK1 3'-UTR or NC. Luciferase activity is presented relative to that of NC and mutant or wild-type ELK1 3'-UTR. (C) ELK1 messenger RNA expression was significantly increased in cervical cancer tissues compared with that in wild-type cervical tissues $\left({ }^{* *} \mathrm{P}<0.01\right)$. ELK1, ETS domain-containing protein Elk-1; miR, microRNA; UTR, untranslated region; NC, negative control.

was further investigated in the CaSki cervical cancer cell line. Following transfection of CaSki cells with scrambled miRNA (negative control), miR-326 mimic or miR-326 inhibitor, the expression level of miR-326 in each group was determined to confirm that the transfection efficiency was satisfactory (Fig. 4A). Following transfection with the miR-326 mimic, the expression level of miR-326 was significantly upregulated ( $\mathrm{P}=0.0005$; Fig. 4A) compared with that in the control group. Following transfection with the miR-326 inhibitor, the expression level of miR-326 was significantly decreased $(\mathrm{P}=0.0190$; Fig. 4A) compared with that in the control group. The mRNA and protein levels of ELK1 in each group were determined using RT-qPCR and western blot analysis, respectively. Upregulation of miR-326 induced a decrease in the mRNA (Fig. 4B) and protein (Fig. 4C) expression levels of ELK1, whereas the downregulation of miR-326 resulted in the upregulation of the mRNA (Fig. 4B) and protein (Fig. 4C) expression levels of ELK1 in CaSki cells. Therefore, the expression levels of ELK1 appear to be negatively regulated by miR-326 in CaSki cells.
ELK1 is able to rescue the phenotypes caused by miR-326 expression. To determine whether ELK1 is the real functional target of miR-326, ELK1 expression plasmids were constructed without the 3'-UTR of ELK1 (pCMV6-ELK1), which are not able to be regulated by $\mathrm{miR}-326$, to perform a rescue experiment. RT-qPCR analysis demonstrated that overexpression of ELK1 is able to restore the ELK1 mRNA level decreased by miR-326 (Fig. 5A). Furthermore, MTT, migration and invasion assays were performed in CaSki and HeLa cells co-transfected with miR-326 mimic plus pCMV6-ELK1 or empty vector, or with negative control plus empty vector. Restoration of ELK1 eliminated the cell viability, invasion and migration that was decreased by miR-326 mimic (Fig. 5B-E). These results demonstrated that $E L K 1$ is a direct functional target gene of miR-326 and that miR-326 functions as a tumor suppressor through ELK1.

\section{Discussion}

Despite the availability of periodic cancer screening, cervical cancer remains one of the most common cancers causing mortality in females (26); therefore, novel approaches to treating the disease are required. In addition to conventional tumor therapeutic regimens, targeted therapies have been developed that interfere with the key signaling pathway components or inhibit the function of tumor-specific factors in cervical cancer (27). Elucidating the underlying molecular mechanism for the progression of cervical cancer will assist with the development of future treatment options for this disease.

miRNAs are considered to be a novel class of regulatory molecules in various human cancers. Changes in miRNA profiling are involved in almost all aspects of cancer biology, including cell proliferation, migration and invasion. Therefore, the development of miRNAs, which are considered to be novel and specific diagnostic and therapeutic tools, has been the subject of numerous studies $(28,29)$.

miR-326 is one of the most upregulated miRNAs in multiple sclerosis and was initially considered to be upregulated in the blood of patients with recurring autoimmune multiple sclerosis (30). A number of studies have demonstrated the involvement of miR-326 in cell apoptosis, invasion, metabolism, proliferation and tumor growth. Wu et al (31) indicated that increased miR-326 could participate in cancer cell apoptosis. In lung cancer, miR-326 regulates cell proliferation and migration (32). Previous studies have demonstrated that miR-326 suppressed tumor growth in medulloblastoma and malignant glioma $(33,34)$. In the present study, the function of miR-326 in cervical cancer was investigated. The aberrant expression of tumor-suppressive or oncogenic miRNAs is able to disrupt normal regulatory mechanisms in cancer cells (35). Therefore, the expression pattern of miR-326 was investigated in human cervical cancer tissues and healthy controls to determine the association between miR-326 expression and cervical carcinogenesis. The expression of miR-326 was decreased in cervical cancer compared with that in wild-type tissues, whereas the overexpression of miR-326 significantly inhibited cell proliferation, migration and invasion in cervical cell lines. The downregulation of miR-326 also significantly promoted cell proliferation, migration and invasion. These 
A

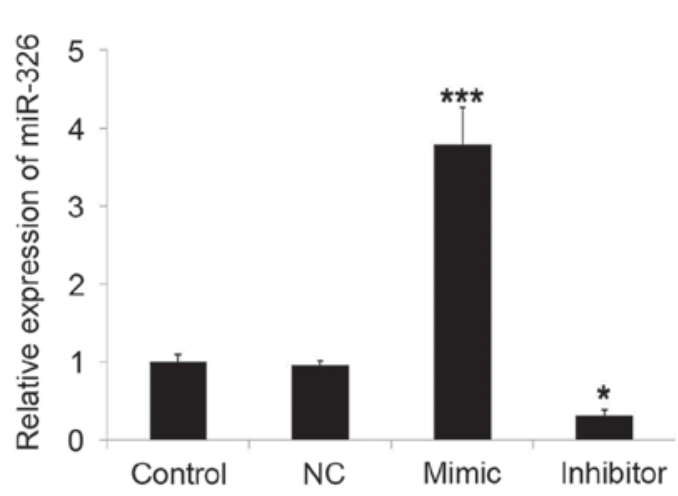

C

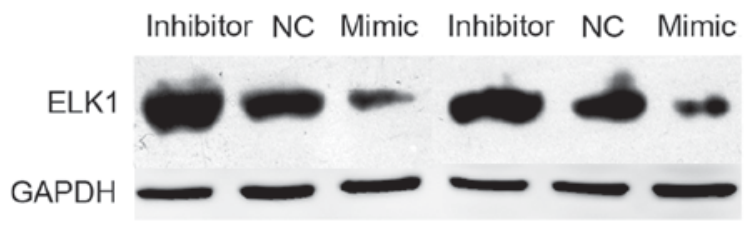

B
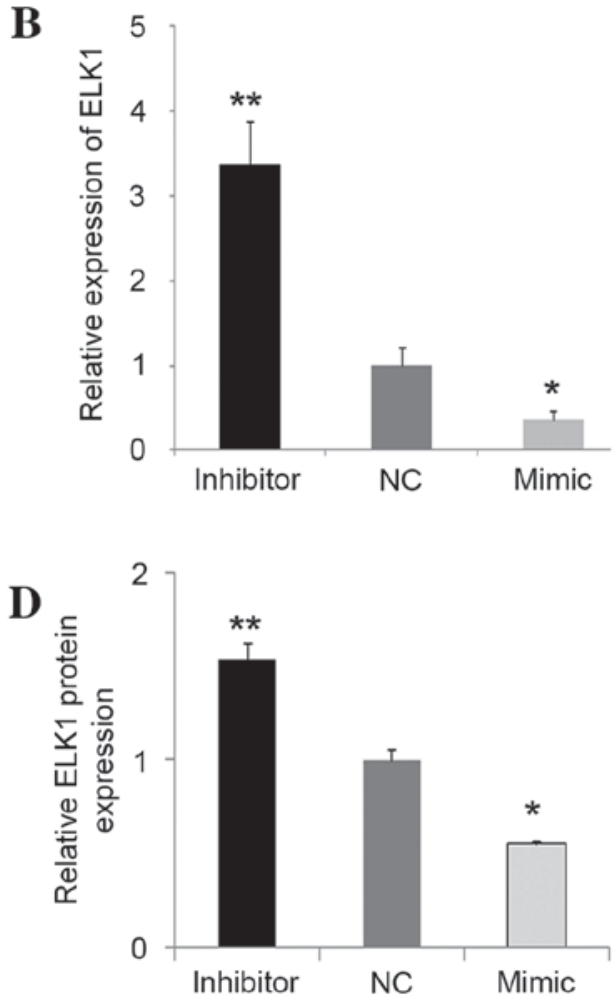

Figure 4. (A) RT-qPCR was used to determine the relative expression level of miR-326 in CaSki cells transfected with scrambled miRNA (NC), miR-326 mimic $\left({ }^{* * * *} \mathrm{P}<0.001\right.$ vs. Control) or miR-326 inhibitor ("P<0.05 vs. Control). Control, untreated CaSki cells. (B) Messenger RNA expression of ELK1 as determined using RT-qPCR following transfection with NC, miR-326 mimic or miR-326 inhibitor. ${ }^{*} \mathrm{P}=0.0137,{ }^{* * *} \mathrm{P}=0.0017$ vs. NC. (C) Protein levels of ELK1 were detected using western blot analysis on CaSki cells transfected with NC, miR-326 inhibitor or miR-326 mimic. GAPDH was used as a loading control. The left three and the right three bands were two sets of independent biological repeats, which exhibited similar trends of ELK1 level in CaSki cells transfected with miR-326 inhibitor, NC or miR-326 mimic. (D) Quantification of the western blot results. Data were normalized to the results using the NC. ${ }^{*} \mathrm{P}=0.0138$, ${ }^{* *} \mathrm{P}=0.005$ vs. $\mathrm{NC}$. RT-qPCR, reverse transcription-quantitative polymerase chain reaction; NC, negative control; miR, microRNA; ELK1, ETS domain-containing protein Elk-1.

results suggest that miR-326 functions as a tumor suppressor, whose upregulation may suppress the progression and metastasis of cervical cancer. Cancer progression and metastasis are associated with the mortality of patients with cervical cancer. Therefore, miR-326 may be a potential target for the treatment of patients with cervical cancer that exhibited upregulated miR-326 expression in tumor tissues.

The underlying molecular mechanism involved in miR326-mediated cervical cancer progression was investigated in the present study, and ELK1 was identified as a potential target gene of miR-326 using bioinformatics analysis. ELK1 contained a miR-326-binding site, and miR-326 was demonstrated to bind directly to the 3'-UTR of ELK1 using a dual-luciferase reporter assay. The overexpression of miR-326 significantly decreased ELK1 expression at the mRNA and protein levels. Significant upregulation of ELK1 mRNA was also observed in cervical cancer tissues, and miR-326 levels were demonstrated to be negatively associated with ELK1 mRNA levels. These results confirmed that miR-326 may partly function as a tumor suppressor by repressing ELK1 expression during the development of cervical cancer.

ELK1, a member of the ETS oncogene family in various types of cancer, functions as a transcription factor that mediates transcriptional regulation using a conserved ETS DNA-binding domain (36). The extracellular-signal-regulated kinase/MAPK signaling pathway serves an important role in numerous biological processes and is frequently deregulated in several disease states, including cancer (37). MAPK signaling primarily contributes to gene expression by activating transcription factors, including ELK1 (38). The activated ELK1 binds to the ELK1 element to regulate target gene expression. A previous study indicated that the ELKI-mediated regulation of the target gene network is involved in actin cytoskeleton regulation and cell migration (38). The important ELK1 target genes in this network partly control cytoskeleton-associated activities, including cell migration (39). Consistent with this previous study, the results of the present study demonstrated that reduced ELK1 expression caused by restoration of miR-326 may be the reason for the suppression of cell migration observed in cervical cell lines. Furthermore, it has been identified that ELK1 was significantly upregulated in cervical cancer tissues (40). Consistent with this previous study, it was also identified in the present study that the expression of $E L K 1$ was considerably upregulated in cervical cancer tissues. Transfection with a miR-326 mimic decreased the luciferase activity and protein expression of ELK1 in cervical cancer cells, indicating that ELK1 may be the target gene of miR-326. Furthermore, rescue experiments were performed using MTT, migration and invasion assays. The results suggested that ELK1 may rescue the suppression effects on proliferation, migration and invasion induced by miR-326 in CaSki and HeLa cells, indicating that miR-326 acts as a tumor suppressor through ELK1. 
$\mathbf{A}$

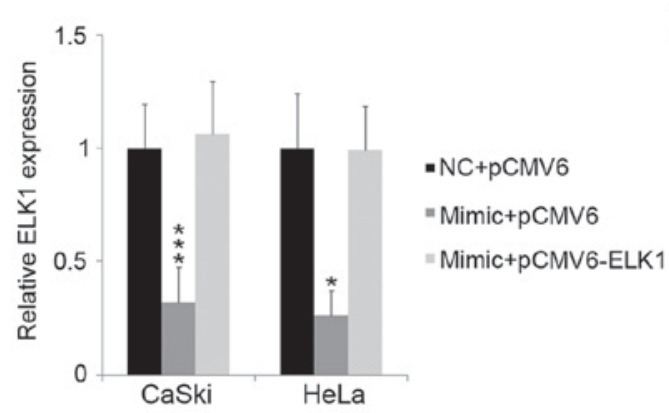

B

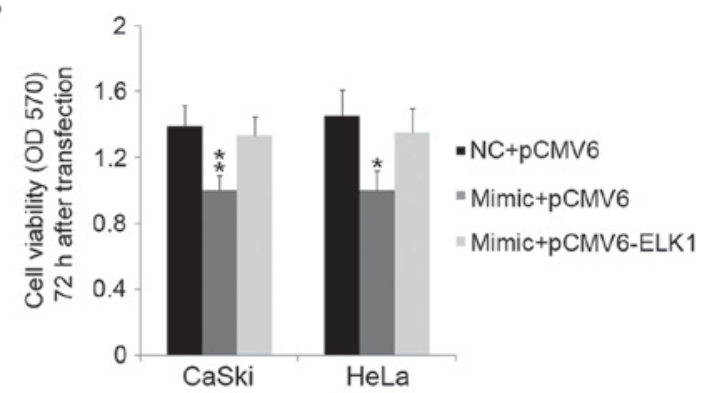

C

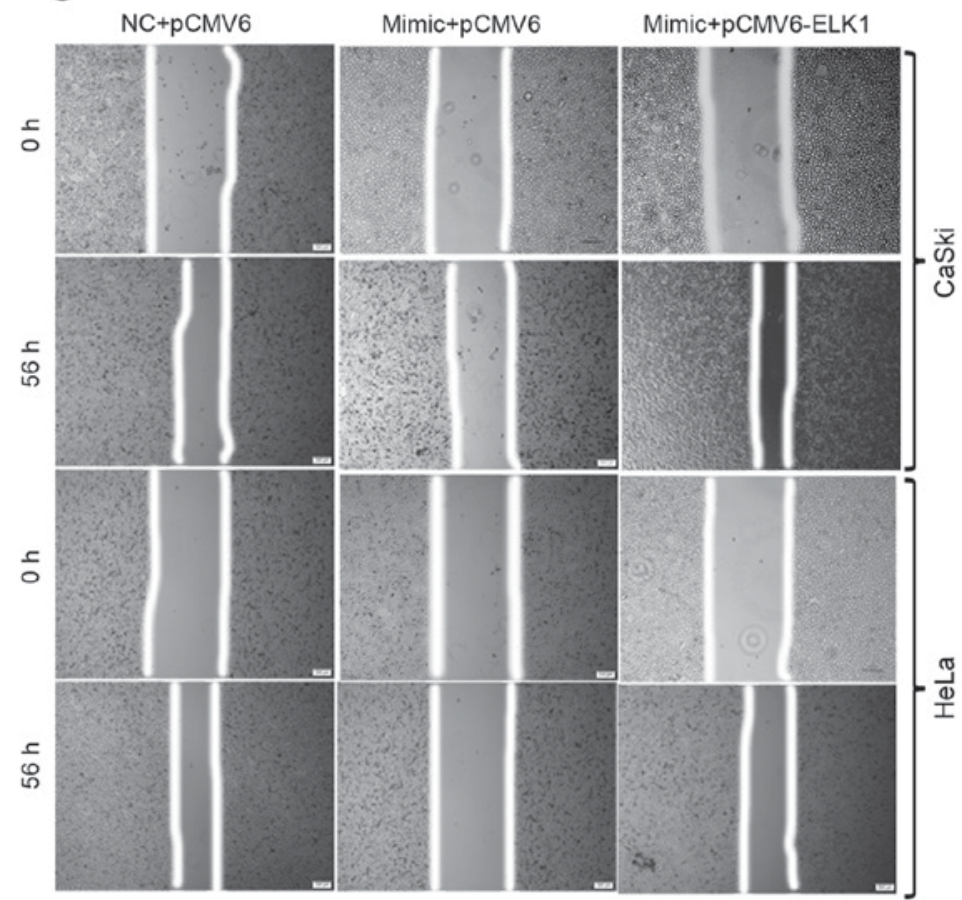

D

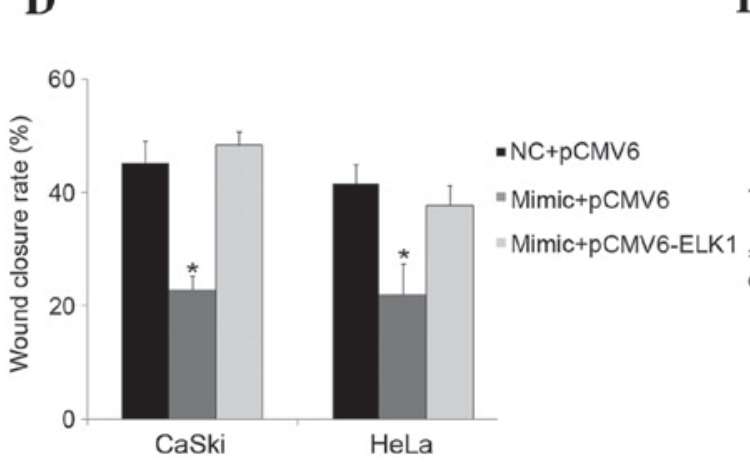

E

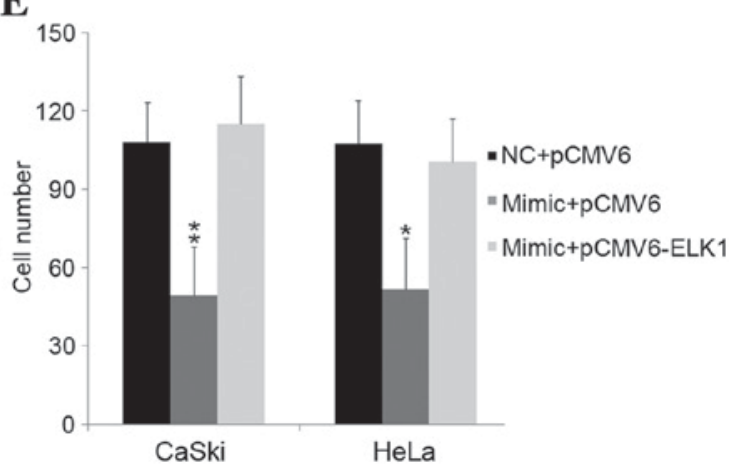

Figure 5. ELK1 abolishes the suppression induced by the miR-326 mimic. (A) Reverse transcription-quantitative polymerase chain reaction was used to confirm that restoration of ELK1 was able to rescue its downregulation caused by the miR-326 mimic. ${ }^{* * *} \mathrm{P}=0.0003,{ }^{* *} \mathrm{P}=0.0015$ vs. NC + pCMV6. Overexpression of ELK1 rescued the suppressive effects of miR-326 on (B) cell viability ( ${ }^{* *} \mathrm{P}=0.0093,{ }^{*} \mathrm{P}=0.017$ vs. NC + pCMV6), (C) A scratch wound-healing assay was performed in CaSki and HeLa cells transfected with NC+pCMV6, mimic+pCMV6 or inhibitor+pCMV6. Magnification, $\mathrm{x} 40$. (D) The migration distance was measured at 0 and $56 \mathrm{~h}$ following scratching of the cells. $\left({ }^{*} \mathrm{P}=0.024,{ }^{*} \mathrm{P}=0.036 \mathrm{vs}\right.$. $\left.\mathrm{NC}+\mathrm{pCMV} 6\right)$ and $(\mathrm{E})$ invasion $\left({ }^{* *} \mathrm{P}=0.0017,{ }^{*} \mathrm{P}=0.012 \mathrm{vs} . \mathrm{NC}+\mathrm{pCMV} 6\right)$. Assays were performed in triplicate.

The results of the present study demonstrate that miR-326 is expressed at lower levels in cervical cancer tissues compared with those in wild-type cervical tissues, and that the overexpression of miR-326 inhibits cell proliferation and invasion. As a novel identified target gene of miR-326,
ELK1 was demonstrated to be upregulated in cervical cancer tissues. These results indicate that the inhibition of miR-326 in cervical cancer may contribute to the malignant phenotype by maintaining high levels of ELK1. Therefore, the identification of miR-326 and its target gene, ELKI, in cervical cancer 
may assist in the understanding of potential molecular mechanisms underlying tumorigenesis and provide novel prognostic markers for the management of cervical cancer.

\section{Acknowledgements}

The present study was supported by the Natural Science Foundation of Shandong Province (grant no. Y2006C44) and the Seed Foundation of the Second Hospital of Shandong University (grant no. 26010275618013).

\section{References}

1. Catarino R, Petignat P, Dongui G and Vassilakos P: Cervical cancer screening in developing countries at a crossroad: Emerging technologies and policy choices. World J Clin Oncol 6 : 281-290, 2015

2. Pimple S, Mishra G and Shastri S: Global strategies for cervical cancer prevention. Curr Opin Obstet Gynecol 28: 4-10, 2016.

3. Morris E and Roett MA: Genital cancers in women: Cervical cancer. FP Essent 438: 18-23, 2015.

4. Karlidag T, Cobanoglu B, Keles E, Alpay HC, Ozercan I, Kaygusuz I, Yalcin S and Sakallioglu O: Expression of Bax, p53, and p27/kip in patients with papillary thyroid carcinoma with or without cervical nodal metastasis. Am J Otolaryngol 28: 31-36, 2007.

5. Wang X, Tang S, Le SY, Lu R, Rader JS, Meyers C and Zheng ZM Aberrant expression of oncogenic and tumor-suppressive microRNAs in cervical cancer is required for cancer cell growth PLoS One 3: e2557, 2008.

6. Noordhuis MG, Eijsink JJ, Roossink F, de Graeff P, Pras E, Schuuring E, Wisman GB, de Bock GH and van der Zee AG: Prognostic cell biological markers in cervical cancer patients primarily treated with (chemo)radiation: A systematic review. Int J Radiat Oncol Biol Phys 79: 325-334, 2011.

7. Davis-Dusenbery BN and Hata A: Mechanisms of control of microRNA biogenesis. J Biochem 148: 381-392, 2010.

8. Garzon R, Calin GA and Croce CM: MicroRNAs in cancer. Annu Rev Med 60: 167-179, 2009.

9. Bartel DP: MicroRNAs: Genomics, biogenesis, mechanism, and function. Cell 116: 281-297, 2004.

10. Bartel DP: MicroRNAs: Target recognition and regulatory functions. Cell 136: 215-233, 2009.

11. Juan L, Tong HL, Zhang P, Guo G, Wang Z, Wen X, Dong Z and Tian YP: Identification and characterization of novel serum microRNA candidates from deep sequencing in cervical cancer patients. Sci Rep 4: 6277, 2014.

12. Karakatsanis A, Papaconstantinou I, Gazouli M, Lyberopoulou A, Polymeneas G and Voros D: Expression of microRNAs, miR-21, miR-31, miR-122, miR-145, miR-146a, miR-200c, miR-221, miR-222, and miR-223 in patients with hepatocellular carcinoma or intrahepatic cholangiocarcinoma and its prognostic significance. Mol Carcinog 52: 297-303, 2013.

13. Zeng K, Zheng W, Mo X, Liu F, Li M, Liu Z, Zhang W and Hu X: Dysregulated microRNAs involved in the progression of cervical neoplasm. Arch Gynecol Obstet 292: 905-913, 2015.

14. Díaz-González Sdel M, Deas J, Benítez-Boijseauneau O, Gómez-Cerón C, Bermúdez-Morales VH, RodríguezDorantes M, Pérez-Plasencia C and Peralta-Zaragoza O: Utility of microRNAs and siRNAs in Cervical Carcinogenesis. Biomed Res Int 2015: 374924, 2015.

15. Wang L, Wang B, Fang M, Guo F and Cui M: Identification of microRNAs and target genes involved in serous ovarian carcinoma and their influence on survival. Eur J Gynaecol Oncol 35: 655-661, 2014.

16. Tarasov VA, Matishov DG, Shin EF, Boiko NV, Timoshkina NN, Makhotkin MA, Lomonosov AM, Kirpii AA, Kit OI and Maksimov AY: Coordinated aberrant expression of miRNAs in colon cancer. Genetika 50: 1232-1244, 2014 (In Russian).

17. Serpico D, Molino L and Di Cosimo S: microRNAs in breast cancer development and treatment. Cancer Treat Rev 40: 595-604, 2014

18. Mo W, Tong C, Zhang Y and Lu H: microRNAs' differential regulations mediate the progress of human papillomavirus (HPV)-induced cervical intraepithelial neoplasia (CIN). BMC Syst Biol 9: 4, 2015
19. Kim J, Krichevsky A, Grad Y, Hayes GD, Kosik KS, Church GM and Ruvkun G: Identification of many microRNAs that copurify with polyribosomes in mammalian neurons. Proc Natl Acad Sci USA 101: 360-365, 2004

20. Thatcher EJ, Flynt AS, Li N, Patton JR and Patton JG: MiRNA expression analysis during normal zebrafish development and following inhibition of the Hedgehog and Notch signaling pathways. Dev Dyn 236: 2172-2180, 2007.

21. Meng F, Henson R, Lang M, Wehbe H, Maheshwari S, Mendell JT, Jiang J, Schmittgen TD and Patel T: Involvement of human micro-RNA in growth and response to chemotherapy in human cholangiocarcinoma cell lines. Gastroenterology 130: 2113-2129, 2006

22. Zhang ZL, Bai ZH, Wang XB, Bai L, Miao F and Pei HH: miR-186 and 326 predict the prognosis of pancreatic ductal adenocarcinoma and affect the proliferation and migration of cancer cells. PLoS One 10: e0118814, 2015.

23. Wang S, Lu S, Geng S, Ma S, Liang Z and Jiao B: Expression and clinical significance of microRNA-326 in human glioma miR-326 expression in glioma. Med Oncol 30: 373, 2013.

24. Zhou J, Xu T, Yan Y, Qin R, Wang H, Zhang X, Huang Y, Wang Y, Lu Y, Fu D and Chen J: MicroRNA-326 functions as a tumor suppressor in glioma by targeting the Nin one binding protein (NOB1). PLoS One 8: e68469, 2013

25. Livak KJ and Schmittgen TD: Analysis of relative gene expression data using real-time quantitative PCR and the 2(-Delta Delta (CT)) method. Methods 25: 402-408, 2001.

26. Janicek MF and Averette HE: Cervical cancer: Prevention, diagnosis, and therapeutics. CA Cancer J Clin 51: 92-118, 2001.

27. Magné N, Deutsch E and Haie-Meder C: Current data on radiochemotherapy and potential of targeted therapies for cervical cancers. Cancer Radiother 12: 31-36, 2008.

28. Iorio MV and Croce CM: microRNA involvement in human cancer. Carcinogenesis 33: 1126-1133, 2012.

29. Garzon R, Marcucci G and Croce CM: Targeting microRNAs in cancer: Rationale, strategies and challenges. Nat Rev Drug Discov 9: 775-789, 2010.

30. Ferretti E, De Smaele E, Miele E, Laneve P, Po A, Pelloni M, Paganelli A, Di Marcotullio L, Caffaelli E, Screpanti I, et al: Concerted microRNA control of Hedgehog signalling in cerebellar neuronal progenitor and tumour cells. EMBO J 27: 2616-2627, 2008.

31. Wu H, Wang Y, Wu C, Yang P, Li H and Li Z: Resveratrol induces cancer cell apoptosis through miR-326/PKM2-mediated ER stress and mitochondrial fission. J Agric Food Chem 64: 9356-9367, 2016

32. Wang R, Chen X, Xu T, Xia R, Han L, Chen W, De W and Shu Y: MiR-326 regulates cell proliferation and migration in lung cancer by targeting phox $2 \mathrm{a}$ and is regulated by HOTAIR. Am J Cancer Res 6: 173-186, 2016.

33. Kefas B, Comeau L, Floyd DH, Seleverstov O, Godlewski J, Schmittgen T, Jiang J, diPierro CG, Li Y, Chiocca EA, et al: The neuronal microRNA miR-326 acts in a feedback loop with notch and has therapeutic potential against brain tumors. J Neurosci 29: 15161-15168, 2009.

34. Zhou J, Xu T, Yan Y, Qin R, Wang H, Zhang X, Huang Y, Wang Y, Lu Y, Fu D and Chen J: MicroRNA-326 functions as a tumor suppressor in glioma by targeting the Nin one binding protein (NOB1). PLoS One 8: e68469, 2013.

35. Galamb Á, Benczik M, Zinner B, Vígh E, Baghy K, Jeney C, Kiss A, Lendvai G and Sobel G: Dysregulation of microRNA expression in human cervical preneoplastic and neoplastic lesions. Pathol Oncol Res 21: 503-508, 2015.

36. Hollenhorst PC, McIntosh LP and Graves BJ: Genomic and biochemical insights into the specificity of ETS transcription factors. Annu Rev Biochem 80: 437-471, 2011.

37. Martinelli E, Morgillo F, Troiani T and Ciardiello F: Cancer resistance to therapies against the EGFR-RAS-RAF pathway: The role of MEK. Cancer Treat Rev 53: 61-69, 2016.

38. O'Donnell A, Odrowaz Z and Sharrocks AD: Immediate-early gene activation by the MAPK pathways: What do and don't we know? Biochem Soc Trans 40: 58-66, 2012.

39. Odrowaz Z and Sharrocks AD: ELK1 uses different DNA binding modes to regulate functionally distinct classes of target genes. PLoS Genet 8: e1002694, 2012.

40. Pallai R, Bhaskar A, Sodi V and Rice LM: Ets1 and Elk1 transcription factors regulate cancerous inhibitor of protein phosphatase $2 \mathrm{~A}$ expression in cervical and endometrial carcinoma cells. Transcription 3: 323-335, 2012. 